\title{
Das Hausdorff Center for Mathematics Bonn
}

\author{
Karl-Theodor Sturm, Koordinator und Clustersprecher, \\ im Gespräch mit Stefan Hartmann
}

Das Hausdorff Center ist nun nicht mehr der einzige mathematische Exzellenzcluster in Deutschland, sondern es gibt vier - neben Bonn noch Berlin, Münster und Heidelberg. Was bedeutet das für Bonn - und für Deutschland?

Die Entscheidung der Exzellenzkommission war eine sehr gute Nachricht. Je mehr Mathematik gefördert wird, desto besser ist das nicht nur für unser Fach, sondern auch für den Technologie- und Wissenschaftsstandort Deutschland. Die anderen Cluster ergänzen unser Profil in teilweise komplementärer Weise. Ohne die innerdeutsche Konkurrenz in irgendeiner Weise schmälern zu wollen: Wir sehen unsere Konkurrenten eher im internationalen Markt. Globalisierung und zunehmende Mobilität von Wissenschaftlerinnen und Wissenschaftlern sind unwiderrufliche Fakten - und fächerübergreifend zählt der Forschungsstandort Deutschland leider nach wie vor zu den Verlierern dieser Entwicklungen. Wir als Hausdorff Center for Mathematics sind fest entschlossen, die Herausforderungen der Globalisierung anzunehmen und sie zu unserem Vorteil zu nutzen.

Was fehlt Bonn denn noch, um auf Augenhöhe mit den weltweiten Top-Universitäten zu sein? Oder ist Bonn das bereits?

In Europa spielen wir sicherlich bereits in der Champions League. Diesen Platz wollen wir sichern und auch weiter ausbauen. Ein Vorteil der ETH beispielsweise war bislang, dass dort klar war, dass ihre Ressourcen auch mittelund langfristig zur Verfügung stehen. Diese Gewissheit hatten wir bis vor kurzem nicht. Durch die langfristig angelegte Förderung der Exzellenzstrategie und durch die enorme Unterstützung der Universität Bonn und des Landes Nordrhein-Westfalen sind wir aber künftig noch stärker aufgestellt und bestens im internationalen Wettbewerb positioniert.

$\mathrm{Zu}$ den Vereinigten Staaten: Ich bin bei der Beurteilung internationaler Rankings vorsichtig, vor allem, was den hohen Anteil US-amerikanischer Hochschulen in den Top 50 angeht. Umgekehrt muss man natürlich sehen, dass die finanziellen Möglichkeiten von Princeton, Harvard und Stanford Lichtjahre von unseren entfernt sind. Das kann man punktuell kompensieren, aber die Unterschiede kann man nicht wegdiskutieren und auch nicht durch gute Organisation und viel Anstrengung vollständig wettmachen. In Einzelbereichen können wir aber hervorragend mithalten mit diesen Top-Universitäten oder sind sogar besser, das haben wir ja diesen Sommer eindrücklich unter Beweis gestellt.

Was zeichnet Bonn als Mathematikstandort aus, was sind Bonns Stärken?

Bonn hat eine gewachsene Struktur in der Mathematik, die einerseits sehr breit angelegt ist, aber andererseits auch durch viele individuelle Leistungsträger besticht. Das sind natürlich zunächst augenfällig die beiden FieldsMedaillisten in der reinen Mathematik, die Bonn international an der Spitze der Forschung vertreten, mit unserem weltweit berühmten Max-Planck-Institut als Marke. Das sind aber auch Leistungsträger aus ganz anderen Bereichen - Partielle Differentialgleichungen, Stochastik, Diskrete Mathematik, Numerische Simulation, Topologie, Darstellungstheorie und viele andere, in denen wir Kolleginnen und Kollegen haben, die weltweit in der allerersten Reihe stehen - und das nicht erst seit wenigen Jahren, sondern aus gewachsenen Strukturen heraus, die systematisch verstärkt wurden. Ganz entscheidend ist, dass sich die vielen exzellenten Gruppen auch gegenseitig befruchten und sich zu einem großen Team zusammengefunden haben. Dies hat eine Attraktivität geschaffen, die weit über den deutschen Markt hinausgeht, und dies wiederum hat in den letzten Jahren verstärkt dazu geführt, dass Bonn sehr viel internationaler geworden ist. Es ist sowohl gelungen, international herausragende Mathematikerinnen und Mathematiker in den Forschungs- und Lehrbetrieb zu integrieren - etwa Sergio Conti, Juan Velazquez oder Massimiliano Gubinelli - als auch umgekehrt führende deutsche Vertreterinnen und Vertreter wie Barbara Niethammer, Peter Teichner oder Christoph Thiele aus dem Ausland nach Deutschland zurückzuholen und insbesondere auch das Abwandern der weltweit umworbenen, besten Köpfe zu verhindern.

Es ist ja sehr viel Geld in den letzten Jahren nach Bonn geflossen durch die Exzellenzinitiative. Was gibt Bonn denn umgekehrt zurück? Was tut das Hausdorff Center für die mathematische Community, vor allem in Deutschland?

Zunächst einmal ist die Art mathematische Grundlagenforschung, die wir machen, mittel- und langfristig ein echter Standortvorteil für Deutschland. Wir haben weiterhin einen Großteil der Fördergelder von Beginn an darauf verwendet, dass sie der mathematischen Community - in Deutschland, aber auch international - zugutekommen, vor allem durch den Aufbau des Hausdorff Research Institute for Mathematics. Wir betrachten dieses Institut, das 


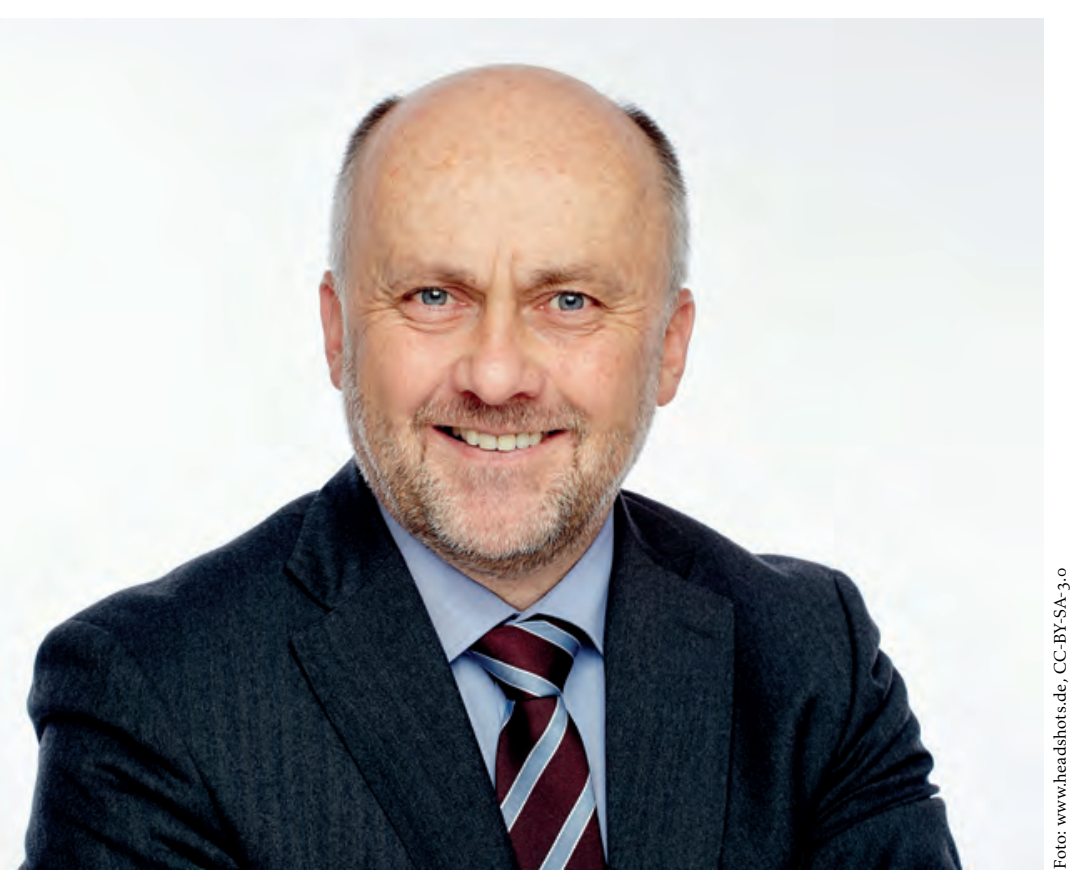

Karl-Theodor Sturm, Koordinator und Clustersprecher des Hausdorff Center Bonn

„HIM“, als eine Serviceeinrichtung für die mathematische Community. Anträge für Programme und Teilnahmen am HIM können aus der ganzen Welt gestellt werden; Bonner Kollegen genießen da keine Vorzugsbehandlung. Die Leitungsstruktur des HIM ist separat von der des HCM. Zudem wird die Programmauswahl von einem internationalen Fachbeirat vorgenommen. Aus geographischen Gründen kommen die Angebote des HIM natürlich besonders auch Mathematikerinnen und Mathematikern in Deutschland zugute - ob das nun Nachwuchswissenschaftlerinnen und -wissenschaftler sind, die kostenlose Fortbildungen bekommen oder etablierte Persönlichkeiten, die die Möglichkeit bekommen, sich in die Programmorganisation einzubringen und vier Monate lang die Forschung in einem speziellen Teilgebiet gemeinsam mit den besten Mathematikerinnen und Mathematikern weltweit voranzutreiben.

Eine weitere Serviceeinrichtung für die mathematische Community, die seit zwei Jahren im Aufbau ist und in der dritten Förderperiode mit Nachdruck ausgebaut werden soll, ist die Hausdorff School. Diese bietet innovative Fortbildungskonzepte für Postdocs an. Eine Form der Weiterbildung, die bislang sehr stiefmütterlich behandelt wurde, sowohl in Deutschland als auch weltweit, und für die wir großen Bedarf sehen. Wir wollen künftig bis zu zehn Mal im Jahr wissenschaftliche Kompaktprogramme in Form von Sommer- oder Winterschulen organisieren. Des Weiteren bieten wir Soft Skill-Seminare wie beispielsweise zu Präsentationstechniken oder Interviewtrainings und ein Mentorenprogramm zur akademischen Lehre an. Die allermeisten dieser Angebote stehen Nachwuchskräften aus der ganzen Welt zur Verfügung, wobei aus geographischen Gründen die Community in Deutschland vermutlich wieder besonders profitiert.
Sie sprechen die Nachwuchsförderung an. Dies war von Beginn an eines der wichtigsten Ziele des Hausdorff Center. Gibt es da noch weitere Angebote des HCM auBer der Hausdorff School?

Hier ist vor allem unsere Bonn International Graduate School of Mathematics zu nennen, die wir von Beginn der Exzellenzförderung an aufgebaut haben. Die Stellen für Promovierende werden - ebenso wie die Postdoc-Stellen und die Nachwuchsprofessuren - nicht einzelnen Professuren zugeordnet, sondern sie werden gebietsübergreifend weltweit ausgeschrieben und kompetitiv - und zwar auch kompetitiv zwischen den Bonner Arbeitsgruppen besetzt. Hier lehnen wir uns an die Verfahren an, wie man sie aus den USA kennt, mit festen jährlichen Ausschreibungen. Die besten Kandidatinnen und Kandidaten sollen die Stellen erhalten, unabhängig von der genauen Fachrichtung. Die BIGS unterstützt die Promovierenden mit optimalen Bedingungen. Sie profitieren von einer unglaublich reichhaltigen Auswahl an fortgeschrittenen Vorlesungen und Forschungsseminaren und sind sehr gut untereinander vernetzt.

Durch die Exzellenzinitiative konnten wir zudem die Bonn Junior Fellow-Stellen schaffen. Diese richten sich an Nachwuchswissenschaftlerinnen und -wissenschaftler, die bereits eine Postdoc-Phase hinter sich haben und begonnen haben, aktuell ihr eigenes Forschungsprogramm zu entwickeln. Wir bieten ihnen W2-Professuren auf Zeit an mit maximaler wissenschaftlicher Freiheit, am ehesten vergleichbar mit dem Emmy Noether-Programm der DFG. Diese attraktiven Stellen werden jährlich ausgeschrieben und in einem sehr ambitionierten Berufungsverfahren vergeben, auf das wir sehr stolz sind. Mit nur drei Monaten zwischen Bewerbung und Ruferteilung stellen wir hier 
Das Hausdorff Center for Mathematics

Adresse

Gegründet

Koordinator

Geschäftsführer

Beteiligte Einheiten

Hauptkomponenten
Endenicher Allee 62, 53115 Bonn. hcm.uni-bonn.de

2006

Prof. Dr. Karl-Theodor Sturm

Dr. Michael Meier

- Mathematisches Institut

- Institut für Angewandte Mathematik

- Institut für Numerische Simulation

- Forschungsinstitut für Diskrete Mathematik

- Gruppe der beteiligten Forscherinnen und Forscher am FB Wirtschaftswissenschaften

- Max-Planck-Institut für Mathematik

- Hausdorff Research Institute for Mathematics

(HIM, him.uni-bonn.de)

Direktor: Prof. Dr. Christoph Thiele

- Bonn International Graduate School of Mathematics

(BIGS, bigs.uni-bonn.de)

Direktorin: Prof. Dr. Barbara Niethammer

- Hausdorff School for Advanced Studies in Mathematics

(HSM, hsm.uni-bonn.de)

Direktor: Prof. Dr. Patrick Ferrari

Bonn Junior Fellow-Programm (BJF-Programm)

25

ca. 50 Professorinnen und Professoren, jeweils ca. 100 Promovierte und Promovierende

Insgesamt zehn Forschungsfelder in drei verschiedenen Sektionen:

A: Deep structures of spaces and invariants

B: High dimensionality, singularities, and randomness

C: Mathematical modeling, analysis, and algorithms

Zudem fünf interdisziplinäre Forschungseinheiten (Interdisciplinary research units, IRUs), darunter je eine mit den Bereichen Materialwissenschaften und Mathematische Physik sowie drei mit dem Bereich Lebenswissenschaften wohl einen bundesdeutschen Rekord auf. Für einige der Stellen gibt es außerdem die Möglichkeit einer Tenure Track-Professur. Auch dieses Programm richtet sich an Bewerberinnen und Bewerber aus der ganzen Welt. Sie müssen übrigens kein Deutsch sprechen. Es ist uns damit gelungen, herausragende Nachwuchswissenschaftlerinnen und -wissenschaftler auch von US-amerikanischen Topuniversitäten zu gewinnen. Und noch etwas Wichtiges: Wir werden mindestens ein Drittel dieser Stellen mit Frauen besetzen. Ein weiteres Instrument zur Nachwuchsförderungen sind die Junior-Trimesterprogramme am HIM mit einer „bottom up"-Struktur. Hier können sich junge Nachwuchswissenschaftlerinnen und -wissenschaftler aus der ganzen Welt bewerben, am liebsten direkt als Gruppe. Diese kleinen Gruppen können mit maximaler Freiheit in Eigenregie ihr Forschungsprogramm über vier Monate gestalten und dabei Top-Leute aus der ganzen Welt einladen. Dieses Konzept ist weltweit einzigartig und sorgt für richtig viel Dynamik in der mathematischen Forschungslandschaft.
Was wird sich in der dritten Förderung ändern? Welche strukturellen Erweiterungen sind geplant? Oder geht es einfach nur so weiter wie bisher?

Eine Sache, die ich schon genannt hatte und die uns sehr wichtig ist, ist die Hausdorff School, die wir mit großem Nachdruck weiter ausbauen möchten.

Des Weiteren haben wir ein weltweites Netzwerk etabliert, das unsere bisherigen internationalen Kontakte weiter stärkt und ihnen eine institutionalisierte Form gibt: das GlobalMathNetwork. Die Kooperationspartner in der derzeitigen Phase sind das Courant Institute in New York, die École Normale Supèrieure in Paris sowie die Universitäten in Kyoto und Peking. All diese Einrichtungen verfügen über hervorragende Promotionsprogramme, kombiniert mit starken Forschungsinstituten in der Mathematik. Der Kern des GlobalMathNetwork besteht darin, dass jede der Institutionen einem Großteil seiner Promovierenden anbietet, mindestens zwei Monate im Ausland zu verbringen und dafür finanzielle Mittel bereitzustellen. Der Aufent- 
halt muss nicht bei einem der Kooperationspartner des GlobalMathNetwork stattfinden, aber wenn es sich um einen Kooperationspartner handelt, dann ist die Unterstützung durch den Gastgeber maximal gewährleistet. Insbesondere fallen dann keine Studiengebühren an.

Die dritte Neuerung, auf die wir sehr viel Wert legen, ist die Stärkung der Zusammenarbeit mit den Nachbarwissenschaften. Wir haben dafür ein neues Programmformat konzipiert, die Interdisciplinary Research Units, kurz IRUs, die an ausgewählten Schnittstellen zu den Nachbarwissenschaften angesiedelt sein werden. Eingebettet in ein Netzwerk von etablierten Wissenschaftlerinnen und Wissenschaftlern von beiden Seiten der beteiligten Fächer wird jeweils eine Nachwuchsgruppe aufgebaut, typischerweise ausgestattet mit einer befristeten W2-Professur und je einer Postdoc- und einer Promotionsstelle. Diese IRUs sollen paritätisch von beiden Kooperationspartnern finanziert werden. Entsprechende Kooperationen wurden vereinbart mit den Materialwissenschaften, mit der Mathematischen Physik und - in Form von sogar drei solcher Gruppen - den Lebenswissenschaften. Im Bereich der Lebenswissenschaften sind die dazugehörigen Berufungsverfahren gemeinsam mit ImmunoSensation, einem weiteren Bonner Exzellenzcluster, schon auf den Weg gebracht und es liegt bereits die erste Rufannahme vor. Professor Hasenauer wird sich mit seiner Arbeitsgruppe „Biomathematik“ auf die Entwicklung von Methoden zu datengetriebenen Modellierungen biologischer Prozesse konzentrieren. Damit kann man biologische Hypothesen überprüfen und zukünftige Experimente maßgeschneidert auswählen.

\section{Was wünschen Sie sich für die Zukunft des Hausdorff} Center?

Zunächst ist mein Wunsch natürlich, dass die Arbeitsbedingungen hier in Bonn nach wie vor gute Mathematik ermöglichen und dass wir die besten Kräfte der Welt ob nun als Gäste oder dauerhaft - zu uns bringen. Weiterhin wünsche ich mir, dass wir bei großen wichtigen Problemen der Mathematik, aber auch bei mathematischen Problemen der Gesellschaft, Wesentliches beitragen in den nächsten Jahren, dass wir ein dynamisches Projekt bleiben, uns also weiterentwickeln und immer wieder neue Schwerpunkte setzen. Wir können nicht die komplette Mathematik abdecken, aber wir sollten unsere Energie so bündeln, dass wir bei allen wichtigen Fragen zur Forschung entscheidend mitwirken.

\author{
Stefan Hartmann \\ Wissenschaftlicher Mitarbeiter Hausdorff Center for Mathematics (HCM), \\ Rheinische-Friedrich-Wilhelms-Universität Bonn \\ Endenicher Allee 6253115 Bonn \\ stefan.hartmann@hcm.uni-bonn.de
}

\title{
Was ist eigentlich die Hausdorff School?
}

\author{
Stefan Hartmann
}

Die Zeit zwischen Promotion und Professur ist eine bislang oft vernachlässigte Übergangsphase. Gerade dort werden die entscheidenden Weichen für die berufliche und private Zukunft gestellt. Die jungen Wissenschaftlerinnen und Wissenschaftler entwickeln nicht nur ihre ersten eigenen Forschungsprogramme einschließlich Personalverantwortung, sondern müssen auch ihre ersten Vorlesungen halten und Seminare leiten - dabei oft ohne jegliche Vorbereitung, geschweige denn systematisches Training. Ohne eine Mentorin oder einen Mentor und ohne die Ausbildung von allgemeinen Lehrkompetenzen gleichen diese Anforderungen für viele einem „Sprung ins kalte Wasser". Die Bonner Hausdorff School for Advanced
Studies in Mathematics möchte diesen Missstand beheben und eine Brücke schlagen zwischen Promotion und Professur, aber auch zu einer Karriere außerhalb der Universität.

Das Angebot der Hausdorff School richtet sich an Postdoktorandinnen und Postdoktoranden weltweit und setzt sich aus mehreren Komponenten zusammen: einem wissenschaftlichen Kursprogramm in Form von Sommeroder Winterschulen, einem Fortbildungsangebot zur Erlangung beruflicher Kernkompetenzen sowie einem Trainingskonzept für die akademische Lehre inklusive eines Mentorenprogramms. Das Kursprogramm besteht aus bis zu zehn einwöchigen Schulen pro Jahr. Das Themenspektrum ist dabei weit gefächert und spiegelt die Mathematik 